Methods A retrospective analysis of 29 SLE patients with PTE admitted to our hospital from January 2009 to August 2018 was conducted.

Results Among the 29 patients, 26 (89.66\%) were female and $3(10.34 \%)$ were male. The age ranged from 23 to 66 years. The SLEDAI scores of 29 cases ranged from 0 to 18 , with scores 15 points in 1 case $(3.45 \%), 10-14$ points in 9 cases (31.03\%), 5-9 points in 11 cases (37.93\%), 0-4 points in 8 cases (27.59\%).Among the 29 patients, 13 patients $(44.83 \%)$ were admitted to the hospital with chest pain or dyspnea as the first symptom. Only one case $(3.45 \%)$ was admitted to the hospital with hemoptysis as the first symptom. Of the 29 patients, $21(72.41 \%)$ had chest pain or difficulty breathing during the course of the disease.Among the 29 patients, 13 cases $(44.83 \%)$ had SLE at the initial diagnosis, and the remaining 16 cases $(55.17 \%)$ had SLE duration ranging from 1 month to 20 years, $3(18.75 \%)$ in one year and 7 $(43.75 \%)$ in 1 to 10 years. The course of disease was more than 10 years in 6 cases $(37.50 \%)$.In this group of patients, 1 case $(3.45 \%)$ with cerebral infarction, 2 cases $(6.90 \%)$ with renal vein thromboembolism, and 14 cases (48.28\%) with lower extremity venous thrombosis. Of the 29 patients, 3 were normal D-D dimers (normal value $0-0.55 \mathrm{mg} / \mathrm{L}$ ), and the remaining $26(89.66 \%)$ were elevated, with an average of $4.50 \mathrm{mg} / \mathrm{L}$.All patients underwent echocardiography, 14 of whom $(48.28 \%)$ indicated pulmonary artery widening. Of the 29 patients, 3 patients did not receive anticardiolipin antibodies, and of the remaining 26, 13 were positive and 13 were negative.In terms of prognosis, 3 of 10 patients (10.34\%) died.

Conclusions SLE combined with PTE is easily missed and misdiagnosed. The clinical manifestations are not typical. In the active stage of SLE, or patients have chest pain and dyspnea symptomsthe, or test indicates that D-D dimer is elevated, or echocardiography indicates pulmonary artery widening, clinicians should think that it might be SLE merge PTE.For patients with anticardiolipin antibody negative, systemic lupus erythematosus should not be relaxed. During the treatment of patients with SLE combined with PTE, they should be alert to the occurrence of thrombosis in other sites.

Funding Source(s): NO

\section{CORTICOSTEROIDS IMPROVE PREGNANCY OUTCOME IN PATIENTS WITH OBSTETRIC ANTIPHOSPHOLIPID SYNDROME}

${ }^{1}$ Leyre Riancho-Zarrabeitia*, ${ }^{2}$ Laura Lopez Marín, ${ }^{3}$ Pedro Muñoz, ${ }^{4}$ Marcos López-Hoyos, ${ }^{5}$ Ana Haya, ${ }^{5}$ Rafael Del Barrio, ${ }^{5}$ Eva Martínez-Díaz, ${ }^{6}$ Victor M Martínez-Taboada. ${ }^{7}$ Hospital Sierrallana; ${ }^{2}$ Rheumatology Department. Hospital Universitario Marqués de Valdecilla; ${ }^{3}$ Servicio Cántabro de Salud; Immunology Department. Hospital Universitario Marqués de Valdecilla; ${ }^{5}$ Ginecology Departments. Hospital Universitario Marqués de Valdecilla; ${ }^{6} \mathrm{Hospital}$ Universitario Marqués de Valdecilla

\subsection{6/lupus-2019-|sm.80}

Background Recurrent pregnancy losses are the most common obstetric manifestation of antiphospholipid syndrome (APS). Despite standard therapy with low dose aspirin (LDA) and low molecular weight heparin (LMWH), the rate of successful pregnancies is not greater than $70 \%$. Corticosteroids have been suggested as a potential therapy in these patients. Our aim is to describe a cohort of patients with obstetric APS treated with low dose corticosteroids.
Methods Retrospective study including 9 women diagnosed with primary APS. Clinical records were reviewed to obtain demographic and clinical data.

Results Two women had a history of thrombosis, while the remaining 7 had merely obstetric manifestations. All had suffered from early pregnancy losses and 1 of them also had a history of fetal death. We studied 42 pregnancies in these women. The mean number of pregnancies was $4.67 \pm 0.71$ per woman. Maternal age was $35.8 \pm 4.6$ years. Overall there were 30 abortions (71.4\%) and 1 fetal death (2.4\%) Regarding treatments used during pregnancy, 25 (59.5\%) pregnancies were on some treatment: LDA (25), LMWH (24), corticosteroids (13), and intravenous immunoglobulins (IVIG) (4). As expected, in all pregnancies treated with corticosteroids, these drugs were combined with LDA and LMWH. When analyzing the effect of therapies, we found a tendency to decrease pregnancy loss in pregnancies treated with LDA (64 vs $82.4 \%$; $\mathrm{p}=0.3)$ and LMWH (62.5 vs $83.3 \% ; \mathrm{p}=0.18)$. Treatment with corticosteroids, significantly increased the rate of successful pregnancy (the rate of pregnancy loss was $38.5 \%$ in treated vs $86.2 \%$ in non treated pregnancies; $p=0.003$ ). The results of the global pregnancy analysis were confirmed by bivariate and multivariate GEE analysis. In the bivariate analysis, LDA tended to be protective $(\mathrm{OR}=0.38$, CI $0.11-1.33 ; \mathrm{p}=0.129)$ and LMWH and corticosteroids significantly protected against pregnancy loss $(\mathrm{OR}=0.34$, CI $0.13-0.85 ; \mathrm{p}=0.021$; and $\mathrm{OR}=0.17$, CI $0.06-0.51 ; \mathrm{p}=0.002$, respectively). After multivariate analysis, only corticosteroids remained inversely associated with pregnancy loss $(\mathrm{OR}=0.09$, CI 0.012-0.683; $\mathrm{p}=0.020$ ). However, the independent effects of LDA/LMWH could not be adequately tested because all patients on corticosteroids were also treated with LDA and LMWH. As adverse events, 2 cases of gestational diabetes and 1 of preeclampsia were observed.

Conclusions The addition of corticosteroids to standard therapy with LDA and LMWH seems to be safe and effective for improving the pregnancy outcome in women with obstetric APS.

Funding Source(s): This work was supported by a next-Val grant from IDIVAL (NVAL 17/19)

\section{$81 \quad$ ANTIPHOSPHOLIPID SYNDROME IN SYSTEMIC LUPUS ERYTHEMATOSUS LEADS TO A MORE SEVERE DISEASE}

'Leyre Riancho-Zarrabeitia*, ${ }^{2}$ Víctor M Martínez-Taboada, ${ }^{3}$ Iñigo Rua Figueroa, ${ }^{4}$ Fernando Alonso, ${ }^{5}$ Maria Galindo Izquierdo, ${ }^{6} J u a n$ Ovalles-Bonilla, ${ }^{7}$ Alejandro OlivéMarqués, ${ }^{8}$ Antonio Fernandez Nebro, ${ }^{9}$ Jaime Calvo, ${ }^{10}$ Javier Narváez-García, ${ }^{11}$ Eva Tomero Muriel, ${ }^{12}$ Esther Uriarte Isacelaya, ${ }^{13}$ Alina Boteanu, ${ }^{14}$ Mariano Andrés, ${ }^{15}$ Mercedes Freire González, ${ }^{16}$ Gregorio Santos Soler, ${ }^{17}$ María E Ruiz-Lucea, ${ }^{18}$ Mónica Ibáñez-Barcelo, ${ }^{19}$ Ivan Castellvi, ${ }^{20}$ Jose Maria Pego Reigosa. ${ }^{1}$ Hospital Sierrallana; ${ }^{2}$ Hospital Universitario Marqués de Valdecilla; ${ }^{3}$ Hospital de Gran Canaria dr. Negrín; ${ }^{4}$ Sociedad Española de Reumatología; ${ }^{5}$ Hospital Universitario 12 De Octubre; ${ }^{6}$ Hospital General Universitario Gregorio Marañón; ${ }^{7}$ Hospital German Trias i Pujol; ${ }^{8}$ UGC de Reumatología, Instituto de Investigación Biomédica de Málaga (IBIMA) Hospital Regional Universitario de Málaga, Spain; ${ }^{9}$ Hospital Universitario Araba; ${ }^{10}$ Hospital de Bellvitge; ${ }^{11}$ Hospital De La Princesa; ${ }^{12}$ Hospital De Donostia; ${ }^{13}$ Hospital Ramón y Cajal; ${ }^{14}$ Hospital General Universitario Alicante; ${ }^{15}$ Hospital Juan Canalejo A Coruña (CHUAC); ${ }^{16}$ Hospital Marina Baixa; ${ }^{17}$ Hospital De Basurto; ${ }^{18}$ Hospital Son Llàtzer De Palma De Mallorca; ${ }^{19}$ Hospital Santa Creu i Sant Pau; ${ }^{20}$ Complexo hospitalario Universitario Vigo

\subsection{6/lupus-2019-Ism.81}

Background Antiphospholipid antibodies (aPL) have been associated with organ damage and certain features in systemic lupus erythematosus (SLE) patients. Our aim was to 